
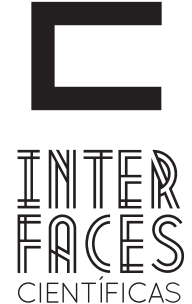

EXATAS E TECNOLÓGICAS

ISSN IMPRESSO - 2359-4934

ISSN ELETRÔNICO - 2359-4942

DOI - 10.17564/2359-4934.2015v1n2p47-56

\title{
SURVEY EM REDES VEICULARES USANDO O MIXIM SOBRE OOMNET++
}

Priscila Copeland Palmeira ${ }^{1}$
Marcos Pereira dos Santos 2

\section{RESUMO}

Este artigo tem por objetivo estudar e expor as definições e bases acerca do protocolo 802.11p, além das ferramentas necessárias, para posteriormente, propor uma condição baseada em redes veiculares, existente em uma rede de comunicação sem fios V2V em larga escala com um funcionamento livre de per- das de pacotes nas redes veiculares VANETs, usando o simulador Ominet++ sobre ele o protocolo 802.11.

\section{PALAVRAS-CHAVES}

Redes veiculares, Survey, Mixim 


\section{ABSTRACT}

This article aims to study and display settings and bases about $802.11 \mathrm{p}$ protocol in addition to the tools needed to subsequently propose a condition-based vehicle networks, existing in a wireless communication network V2V large-scale free operation

\section{RESUMEN}

Este artículo tiene como objetivo estudiar y mostrar la configuración y las bases sobre el protocolo 802.11p y las herramientas necesarias para proponer posteriormente a las redes de transporte basadas en la condición, existente en una red de comunicación inalámbrica V2V gran escala con una operación libre de la pérdida of packet loss in vehicular networks VANETs using Ominet ++ simulator on it the 802.11 protocol.

\section{KEYWORDS}

Vehicular Networks. Survey. Mixim.

de paquetes en redes vehiculares VANETs usando simulador Ominet ++ en él el protocolo 802.11.

\section{PALABRAS CLAVE}

redes vehiculares, Encuesta, Mixim 


\section{INTRODUCÃ̃O}

Com o aumento da população e do desenvolvimento industrial em todo o mundo, o tráfego está ficando cada dia mais pesado, e, assim, aumenta a probabilidade de acidentes de trânsito.

Portanto, o grupo de trabalho IEEE 802.11 elaborou o IEEE 802.11p para apoiar Sistemas Inteligentes de Transporte (ITS). Estas aplicações incluem a troca de dados entre veículos em toda infraestrutura rodoviária, coberta com sua banda licenciada de $5,9 \mathrm{GHz}$ (5,85-5,925 GHz). 0 objetivo do protocolo IEEE 802.11p é fornecer um conjunto mínimo de especificações necessárias, a fim de garantir a interoperabilidade entre dispositivos sem fio, permitindo uma comunicação rápida em situações em que as operações devem ser concluídas em prazos muito mais curtos do que o mínimo possível com infraestrutura ou ad hoc 802.11.

Na última década, ocorreram grandes avanços no campo das redes veiculares ad hoc (VANETs), que são amplamente reconhecidas como um elemento essencial para os Sistemas Inteligentes de Transporte (STIs). Estas relacionam-se com a segurança em suas aplicações, como a pré-colisão para detecção de colisão ou a cooperativa para um aviso prévio, demandando exigências estritas sobre o desempenho dos protocolos de comunicação subjacentes. Por exemplo, a latência de ponta a ponta para máximo de pré-detecção de colisão das aplicações, tem de ser limitada a menos de 20 milissegundos [DER-JIUNN DENG, HSIN-CHIN CHEN, HAN-CHIEH CHAO E YUEH-MIN HUANG, 2010].

As redes ad hoc (VANETs), recentemente começaram a chamar a atenção de muitos pesquisadores na indústria e no âmbito acadêmico. A Comissão Federal de Comunicação (FCC), nos Estados Unidos, alocou a faixa de 5,850-5,925 GHz, para promover comunicações sem fio para rodovias seguras e eficientes. Esta banda está prevista para ser usada no padrão emer- gente de rádio para Dedicated Short Range Communications (DSRC) (ABDRABOU e WEIHUA, 2011).

No entanto, é complexa e desafiadora para alcançar estes requisitos de comunicação, devido a um grande número de efeitos adversos nas características das VANETs, por exemplo, multi-caminho, desaparecimento e sombreamento no canal sem fio, flutuação da densidade de nós em diferentes cenários, e de rede em rápidas mudanças topologias.

Esforços têm sido feitos na comunidade para alcançar rápido e comunicação confiável em ambientes veiculares. Para exemplo, IEEE 802.11p (DER-JIUNN DENG, HSIN-CHIN CHEN, HAN-CHIEH CHAO e YUEH-MIN HUANG, 2010) foi recentemente aprovado como a camada MAC para a comunicação de veículos. No entanto, estudos de avaliação abrangentes mostram que o desempenho $802.11 \mathrm{p}$, incluindo a taxa de perda de pacotes, a probabilidade de colisão, e canal de acesso começa a deteriorar-se como o aumento da densidade de carga ou de tráfego da rede.

Em primeiro lugar, uma vez que é impossível utilizar o RTS / CTS mecanismo para alcançar um 2-hop zona livre de contenção em um cenário de transmissão, 802.11p é muito vulnerável e evita a existência de colisões (PALAN e KHADILKAR, 2011). Para realização desse controle é necessário utilizar o intervalo e prioridade do BEACON bem como a prioridade dos dados.

\section{TRABALHOS RELACIONADOS}

Segundo Der-Jiunn Deng, Hsin-Chin Chen, Han-Chieh Chao e Yueh-Min Huang (2010) os resultados das suas simulações mostram que a proposta de regime não pode apenas aumentar o rendimento canal viável do protocolo legado em mais de $15 \%$, mas também reduzir o atraso de acesso médio de pacotes do protocolo legado, pelo menos, $5 \%$ e a probabilidade 
de colisões de pacotes, no máximo, 60\% em ambientes VANET congestionadas.

Já Peksen e Acarman (2012) chamam atenção sobre as soluções promissoras no sentido de colaboração de infraestrutura e segurança ativa dos veículos rodoviários. Dados de segurança ativos de divulgação em veículo-veículo (V2V) de comunicação requerem a transmissão confiável e em tempo real. Radio difusão e retransmitir as mensagens de segurança para alertar os veículos circundantes sobre situações críticas de condução é considerada a reação que dever ser realizada dentro do tempo ou prazo adequado.

Dentro da análise de Ananthanarayanan e Furse (2011), há necessidade de ter cuidados com antenas que emitem os sinais de ondas eletromagnéticas, vez que um algoritmo aleatório de busca visa otimizar a capacidade para matrizes com amplamente contagem de elementos divergentes, tipo de elemento, combinando diretividade, alinhamento de polarização, a eficiência, a correlação espacial e acoplamento.

A polarização remendo ágil proporciona a melhor capacidade para locais perto do teto da aeronave, enquanto os PIFAs com mais variedade em forma (formas espirais) oferecem a melhor capacidade para locais próximos ao chão. Isto é, porque os sinais que atingem o telhado e os lados mais contêm diversidade de polarização do que os do centro do corpo em que o chão não condutor está localizado.

Conforme Ning Wen e Berry (2006) para que um sistema seja rápido nas transições das informações para o número de veículos possível é necessário que exista uma localização de dados baseados em médias de controle de acesso (MAC), que são os dados em que os veículos transmitirem informações quando eles dirigem por meio de áreas de transmissão de dados.

Ainda, é necessário estudar a informação propagação ao abrigo destes protocolos, para uma simples unidimensional rede de transporte. Especificamente, o cálculo da média de tempo para uma mensagem para propagar uma dada distância e a mensagem de uma probabilidade de atingir essa distância.

O trabalho de Chengyang Wu e outros autores (2012) fala a respeito das redes veiculares que usam o 3G, mostrando que a justiça de cuidar dos interesses de todos os nós de veículos vai ser considerada. Abdrabou e Weihua (2011) falam que o protocolo deve prever a evolução da rede topologia e calcular a probabilidade de colisão de certos nós transmitir simultaneamente.

Mensagens de reserva são trocados entre os nós que podem entrar em conflito uns com os outros, e disputas eventualmente, são resolvidos. 0 trabalho compara o desempenho de o protocolo, medido em termos de probabilidade de colisão, média atraso de acesso ao meio, e razão de entrega de pacotes com 802.11p por simulação. Os resultados mostram que com o custo de maior tempo médio atraso de acesso ao meio, a probabilidade de colisão do protocolo é consideravelmente inferior sobre o 802.11p em vários cenários simulados. Em Palan e Khadilkar (2011) foi utilizado o MixiM no desenvolvido para simulações sem fio e móvel sobre o OMNeT++.

\section{SISTEMAS INTELIGENTES DE TRANSPORTE}

Nos dias de hoje, é possível notar um alto nível de saturação no tráfego mundial, em decorrência do crescente número de veículos nos últimos cinquenta anos. Essa situação afeta diretamente na vida da sociedade, principalmente aquelas que vivem em áreas urbanas, onde as pessoas precisam cada vez mais se deslocar rapidamente. Os resultados principais são: trafego congestionado, acidentes, atrasos e grandes dissipações de poluentes, provindo dos veículos.

Muitas soluções foram propostas, com intuito de reduzirem esses problemas. Contudo, a maioria deles, como por exemplo, a construção de mais e melhores estradas e rodovias, possuem valores muito elevados, além de po- 
derem causar impacto ambiental. Mesmo com essas desvantagens, o desenvolvimento da infraestrutura de transporte é essencial para o crescimento econômico.

Essas dificuldades motivaram a comunidade acadêmica para voltar sua atenção para a área dos Sistemas Inteligentes de Transporte (STI). Os STIs têm como função o desenvolvimento de novos sistemas capazes de resolverem os problemas anteriormente citados. Dessa forma, com a ajuda dos STIs, os sistemas de controle de rodovias podem ser mais seguros, mais eficientes, além de menos agressores ao meio ambiente.

Os STIs envolvem pesquisas entre múltiplas áreas, como, por exemplo, eletrônica, controle, comunicações, robótica, processamento de sinais, e sistemas de informação. Por possuir essa multidisciplinariedade, a complexidade desses sistemas aumenta exponencialmente, o que requer o compartilhamento de conhecimento e cooperação entre diferentes áreas de pesquisa.

Resumidamente, pode-se dizer que os STIs (Figura 1), envolvem os utilizadores, as vias de comunicação (estradas), e os veículos, utilizando-se desses componentes para criar tecnologias apropriadas, que possam tornar as rodovias, veículos e usuários mais inteligentes.

Figura 1 - Componentes do STI

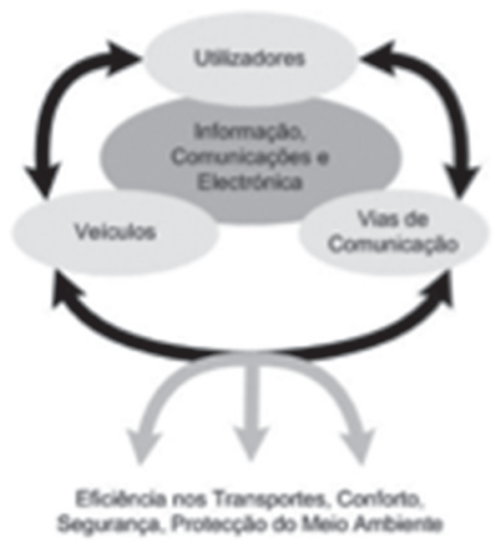

Fonte: Figueiredo (2005).
Tendo em vista o potencial de revolucionar a experiência ao dirigir e a segurança, a comunicação entre veículos (V2V - Vehicle-to-Vehicle), está se tornando cada vez mais popular e tem atraído a atenção da indústria automobilística e da academia, além de permitir a existência de redes veiculares (KARAGIANNIS, ALTINTAS, EKICI, HEIJENK, JARUPAN, LIN, e eweil, 2011).

\section{REDES VEICULARES}

Comunicação entre veículos é uma das partes mais importantes dos STIs, dessa forma, para garantir uma maior segurança, as redes veiculares, utilizam-se dos STIs, além de serviços de assistência aos usuários. As redes veiculares são diferenciadas das outras redes sem-fio pela natureza dos nós, sendo estes os automóveis, os quais possuem interfaces de comunicação sem fio e por dispositivos distribuídos nas estradas e rodovias.

Esse tipo de rede possui alta complexidade e grandes desafios para serem adotadas em larga escala, pois a mobilidade dos nós e dos cenários, o número de nós, além da perda de conectividade e de pacotes durante a transmissão dos dados, dificultam sua projeção.

As redes veiculares estão divididas em três tipos de arquiteturas, sendo elas, ad hoc (vehicular ad hoc NetWORK - VANET), infraestruturada e híbrida (ALVES, CAVALCANTI, CAMPISTA, COSTA, RUBINSTEIN, AMORIM E DUARTE, 2008).

Vehicular ad hoc network (VANET) é um tipo de rede sem-fio, onde cada nó é um veículo em movimento na estrada. Esse tipo de nó se comporta como um roteador transmitindo uma mensagem para outro nó. Essa rede possui dois tipos de comunicação, sendo eles, veículo-para-veículo (V2V) Figura 2, e veículo-para-dispositivo fixo na estrada (V2I). A função primordial de uma VANET é prover aplicações seguras e em tempo real para os usuários. Entregando, dessa forma, os dados no tempo certo, reduzindo acidentes e atrasos. 
A Figura 2 mostra um exemplo clássico de uma VANET. Ela apresenta os dois tipos de comunicação possíveis: V2V e V2I (veículo se comunicando com a RSU). A Figura, também, apresenta um evento de emergência (colisão entre veículos) que as VANETs se encarregam de transmitir para outros veículos para evitar novas colisões.

Figura 2 -VANET

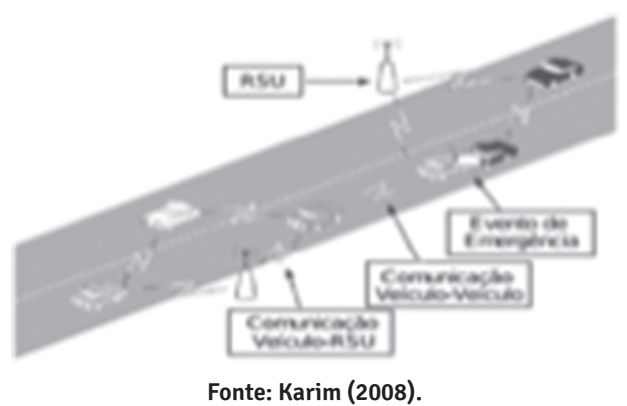

Um dos problemas encontrados é descobrir um meio de fazer as transmissões de dados de forma robusta e eficaz entre VANETs, pois o cenário de trafego urbano possui muitas variáveis, além da mobilidade constante dos nós, das variações de velocidades entre os nós, das segmentações das redes e dos atrasos (SOFRA ET AL., 2011). Podendo assim enviar dados por rotas inexistentes, fazendo com que os dados sejam retransmitidos, ocasionando atraso e perdas (BISHT, KUMAR e MISHRA, 2012).

Em relação à padronização das redes veiculares, em 1999, a Federal Communications Commission (FCC) alocou $75 \mathrm{MHz}$ do espectro de frequências, na faixa de $5,9 \mathrm{GHz}$, para aplicações DSRC (Dedicated Short Range Communications), ilustrada na Figura 3. Essa faixa ela está definida como livre, contudo é paga.
Figura 3 - Faixa de Frequências DSRC

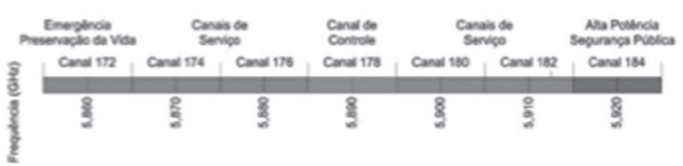

Fonte: Alves, Couto, Campista, Moraes, Rubinstein, Costa, Duarte e Abdalla (2009).

Desde então, o IEEE vem desenvolvendo o padrão Wireless Access in a Vehicular Environment (WAVE), também conhecido como 802.11p, para a camada física de dispositivos móveis que implementam VANETs. Entretanto, o desafio desse protocolo é implementar uma camada MAC que suporte a alta velocidade dos nós, tendo latência minimizada. Foi proposto, também, o padrão $802.11 \mathrm{p}$, o qual define as regras de controle e de acesso físico ao meio.

\section{PROTOCOLO 802.11P}

Uma das padronizações do IEEE foi a arquitetura WAVE ou padrão 802.11p.

O padrão IEEE 802.11p define as camadas físicas e de controle de acesso ao meio (MAC) para redes veiculares e é baseado no padrão de redes locais IEEE 802.11a.

A camada física do IEEE $802.11 p$ realizou três modificações principais em relação ao IEEE 802.11a (JIANG e DELGROSSI, 2008). A primeira, no IEEE 802.11p os canais têm largura de $10 \mathrm{MHz}$ em vez dos $20 \mathrm{MHz}$ definidos no IEEE 802.11a (canais mais estreitos compensa-se o maior espalhamento do atraso RMS esperado em ambientes veiculares). A segunda modificação refere-se aos requisitos de desempenho do receptor de rádio mais restritivos que no IEEE 802.11a (rejeição de canais adjacentes, menor interferência). A terceira modificação especifica a utilização dos rádios IEEE 802.11p nos EUA, no espectro DSRC, define quatro máscaras de transmissão dedicadas a quatro classes de operação distintas. 
O padrão WAVE pode ser usado tanto para comunicação de segurança quanto para aplicações que não envolvam segurança às redes dos veículos, utilizando conceitos de múltiplos canais (CAVALCANTI e RODRIGUES, 2008).

O padrão WAVE é composto pelas seguintes definições, podendo ser visualizado na Figura 4:

- IEEE 1609.0: descreve o framework IEEE 1609 da arquitetura WAVE.

- IEEE 1609.1: especifica serviços e interfaces do RM (gerenciador de recursos) que normalmente é executado na unidade RSU e fornece interoperabilidade entre as aplicações WAVE.

- IEEE 1609.2: especifica a segurança de processamento e formatos das mensagens, lidando com questões relacionadas com autenticação e criptografia.

- IEEE 1609.3: especifica os serviços das camadas LLC (logical link control), de rede e de transporte. Define o protocolo WSMP.

- IEEE 1609.4: especifica a operação de múltiplos canais.

Figura 4 - Pilha de Protocolos WAVE

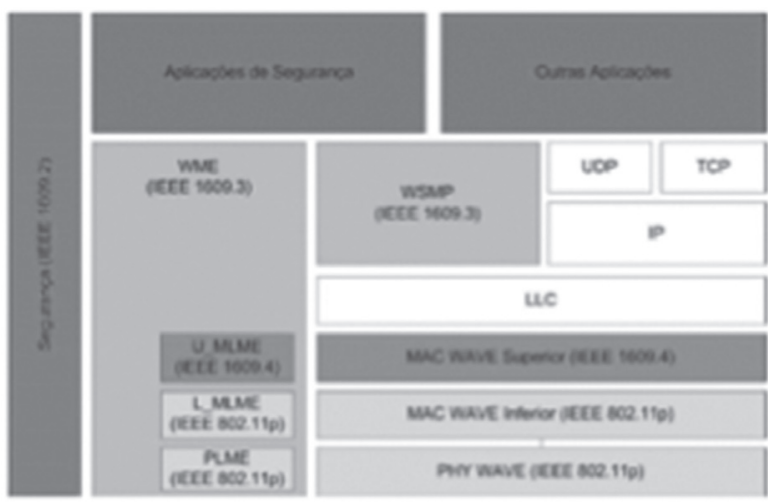

Fonte: Alves, Couto, Campista, Moraes, Rubinstein, Costa, Duarte e Abdalla (2009).

\section{ARQUITETURA DA FERRAMENTA DE SIMULAÇ̃̃O}

O OMNeT++é uma ferramenta de simulação baseada em eventos. Esta não é uma ferramenta de simulação de nenhuma área específica de conhecimento, mas por possuir uma alta flexibilidade, pode ser usada para simular modelos de diversas áreas de conhecimento. Particularmente, tem sido muito utilizada pela comunidade científica para simular redes de computadores e de comunicação de dados.

O OMNeT++ faz uso de uma arquitetura modularizada. Sendo que esses módulos são programados em $\mathrm{C}++$, os quais modelam o comportamento de componentes discretos do sistema no qual se deseja simular. A combinação desses módulos é feita com o uso de uma linguagem de alto nível chamado NED, formando módulos compostos, que por sua vez, podem ser combinados em novos módulos compostos, hierarquicamente (USP, 2012).

Por não se encaixar em apenas uma área de conhecimento, o OMNeT++ permite que suas frameworks sejam desenvolvidas e utilizem seu ambiente de simulação. Estas frameworks são agrupamentos de módulos que simulam uma determinada área de conhecimento. Por exemplo, existe uma framework chamada MiXiM, que agrega ao $O M N e T++$ modelos de simulação de redes, usando o protocolo 802.11p, assim como o SUMO que agrega movimento aos objetos da simulação.

Estas framewors serão utilizadas nas simulações do artigo posterior, que será proposto neste artigo. Quando se usa uma framework, é possível criar simulações sem que se seja necessário programar em C++, já que os módulos que simulam o comportamento dos componentes de um modelo já foram criados. Resta ao usuário o papel de criar topologias e configurações, além de analisar os resultados das simulações. A Figura 5 abaixo ilustra o detalhamento da arquitetura do OMNeT++[USP 2012]. 
Figura 5 - Arquitetura do Simulador OMNeT++

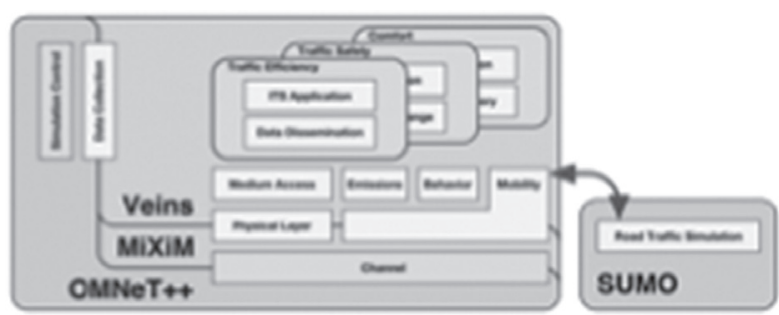

Fonte: USP (2012).

O MixiM é um módulo integrado ao OMNeT++ que pode ser denominado de "simulador misto" e foi desenvolvido para redes sem fio e simulações móveis sobre - OMNeT++. Ele fornece modelos detalhados do canal sem fio (fading), da estimativa de interferência, da conectividade sem fio, e dos modelos de mobilidade com velocidade constante. Também fornece modelos para os obstáculos e muitos dos protocolos de comunicação, principalmente a nível Medium Access Control (MAC). Além disso, ele fornece uma representação gráfica de fácil utilização de redes sem-fio e móveis, e suporte à depuração. 0 MixiM possui uma caixa de ferramentas rica, com a qual o usuário pode simular e estudar a análise de redes sem fio de alto desempenho.

Uma característica do MixiM é esconder a complexidade das simulações entregando ao usuário uma interface transparente e de fácil usabilidade (PALAN e KHADILKAR, 2011). A maioria dos simuladores fornece única frequência para as antenas do sistema, porém o MixiM possui uma rica biblioteca de protocolos e módulos que apóiam a infraestrutura da rede. 0 MixiM pode suportar simulação de redes constituídas até 1000 nós (PALAN e KHADILKAR, 2011), o módulo nó é como mostrado na Figura 6.

Cada nó do módulo é composto constituído de camada de aplicação, rede camada, camada física e camadas MAC. Estas camadas estão ligadas por meio de portas de acesso, sendo que um par de portas é para cima e outro para baixo, possibilitando a passagem das mensagens de dados e mensagens de controle entre os nós e dois pares é usado para trocar mensagens de controle entre nós. É válido salientar que o MAC e a camada física são agrupados em NIC (Módulo de rede placa de interface de cartão) (PALAN e KHADILKAR 2011]. Um nó com uma placa IEEE 802.11 é usado para esta simulação.

Figura 6: a) Exemplo de um nó no MiXiM. b) Exemplo de um Módulo no MiXi

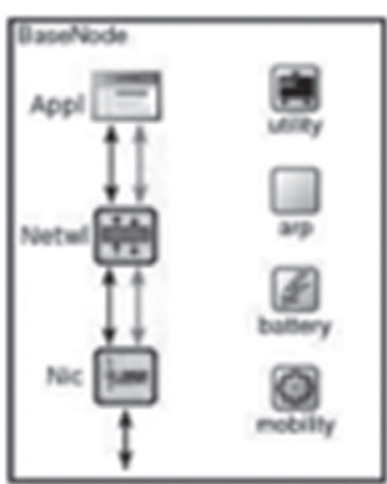

(a)

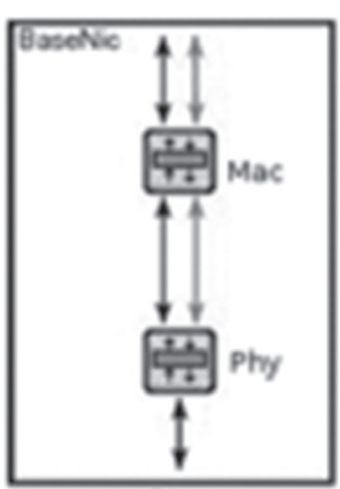

(b)
Fonte: M. Palan e Khadilkar (2011).

Maiores informações sobre o OMNet++ e o MiXiM podem ser encontradas em (MiXiM Project, 2007; Xian et al., 2008; Varga e Hornig, 2008; OM-NeT++, 2011).

\section{CONCLUSÕES}

As redes veiculares possuem uma gama de assuntos que podem ser abordados no âmbito acadêmico. 0 padrão IEEE 802.11p vem se desenvolvendo a cada nova pesquisa. Para se ter uma rede em pleno funcionamento é preciso analisar e entender o tráfego de dados em relação às aplicações das comunicações veiculares. Diante dessas características, assim como da mobilidade dos nós, dinamismo e da conectividade da rede, o roteamento em ambientes veiculares, torna-se uma tarefa complexa.

Neste trabalho, foram discutidas as definições e embasamento teórico para que no trabalho futu- 
ro, possa-se fazer a implementação no OMNet++, em conjunto com o MiXiM, para análise de redução da perda de pacotes em redes VANETs baseadas no protocolo 802.11p. Está ferramentas foram escolhidas por possuírem interface amigável, e ter suporte a vários protocolos de rede, programável, além de seus resultados que podem ser compatíveis com outros softwares, como o R, Scilab e o Matlab.

\section{REFERÊNCIAS}

ABDRABOU, A.; ZHUANG, Weihua, Probabilistic Delay Control and Road Side Unit Placement for Vehicular Ad Hoc Networks with Disrupted Connectivity, In: Selected Areas in Communications, IEEE Journal, v.29, Issue: 1, 2011.

ALVES, R. S., et al. Uma análise experimental da capacidade de redes ad hoc veiculares, In: Simpósio Brasileiro de Telecomunicações (SBrT), 2008.

ALVES, R.S.; et al. Redes Veiculares: Princípios, Aplicações e Desafios, In: $27^{\circ}$ Simpósio Brasileiro de Redes de Computadores e Sistemas Distribuídos, 2009.

ANANTHANARAYANAN P.R.S.; FURSE, C.M., Antenna optimization for vehicular environments, In: Antennas and Propagation (APSURSI), IEEE International Symposium, 2011.

BISHT, A., KUMAR, B., and MISHRA, S. Efficiency evaluation of routing protocols for vehicular ad-hoc networks using city scenario, In: International Conference on Computer Communication and Informatics, 2012. p.1-7.

CAVALCANTI, E RODRIGUES, S. Veer: um algoritmo de seleção de pares em redes ad hoc veiculares. 2008. Dissertação (Mestrado em Engenharia Elétrica) - Universidade Federal do Rio de Janeiro, Rio de Janeiro, RJ - Brasil, 2008.
CHENGYANG Wu, et al. Dynamic cluster based price control and gateway management for VANETs, Communications (ICC), IEEE International Conference, 2012.

DER-JIUNN, Deng; HSIN-CHIN, Chen; HAN-CHIEH, Chao; Yueh-Min, Huang, A Collision Alleviation Scheme for IEEE 802.11p VANETs, Wireless PersCommun - abr. 2010.

DIAS, J. F. Mobilidade em comunicações veiculares. NAP Instituto de Telecomunicações, 2012.

FIGUEIREDO, L., JESUS, I. MACHADO, J. A. T., FERREIRA, J. R. E CARVALHO, J. L. M. Towards the development of intelligent transportation systems, Intelligent Transportation Systems Conference Proceedings - Oakland (CA) USA, 2001.

FIGUEIREDO, L.M.B. Sistemas Inteligentes de Transporte. 2005. Tese (Doutorado em Engenharia Eletrônica e de Computadores) - Universidade do Porto, Porto-Portugal, 2005.

JIANG, D. E DELGROSSI, L. IEEE 802.11p: Towards an international standard for wireless access in vehicular environments, IEEE Vehicular Technology Conference (VTC-Spring), 2008. p.2036-2040.

KARAGIANNIS, G., et al. Vehicular networking: A survey and tutorial on requirements, architectures, challenges, standards and solutions. IEEE Communications Surveys and Tutorials, 2011. 13(4):584-616.

KARIM, R. Vanet: Superior system for content distribution in vehicular network applications. Rutgers University, Department of Computer Science, Tech. Rep, 2008.

KUCUK, K.; KAVAK, A.; YIGIT, H. A Smart Antenna Module Using OMNeT++ for Wireless Sensor Network Simulation. Wireless Communication Systems, 2007. ISWCS 2007. 4th InternationalSymposium. 
MENDONÇA, T. F. VANETs: VehicularAd-Hoc Networks. Instituto de Matemática e Estatística, Universidade de São Paulo-USP, 14 de julho de 2012.

NING, Wen; BERRY, R. Information propagation for location-based MAC protocols in vehicular networks information. Sciences and Systems, 2006 40th Annual Conference.

PALAN, N. G.; KHADILKAR, Aditi. P., Media access control protocol modelling for mobile sensor network-using OMNeT++ -MiXiM network simulator. Sustainable Energy and Intelligent Systems (SEISCON 2011), International Conference.
PEKSEN, Y.; ACARMAN, T. Multihop safety message broadcasting in VANET: A distributed medium access mechanism with a relaying metric. Wireless Communication Systems (ISWCS), 2012 International Symposium.

SOFRA, N., GKELIAS, A., AND LEUNG, K. Route construction for long lifetime in vanets. IEEE Transactions on Vehicular Technology, 2011.

UNIVERSIDADE de São Paulo. Roteiro OMNeT. Disponível em: <http://www.lcs.poli.usp.br/ jra/ptc2460/ OMNET/roteiro_omnet.pdf>. Acesso em: set. 2012. 